\title{
Adenosquamous carcinoma arising from a thyroglossal duct cyst: A case report
}

\author{
YU-SUNG CHANG ${ }^{1}$, HSIN-HAO $\mathrm{SU}^{1}$ and SZU-PEI HO ${ }^{2}$ \\ Departments of ${ }^{1}$ Otolaryngology, and ${ }^{2}$ Pathology and Laboratory Medicine, Kaohsiung Veterans General Hospital, \\ Kaohsiung 813, Taiwan, R.O.C.
}

Received December 23, 2014; Accepted January 26, 2016

DOI: $10.3892 / \mathrm{ol} .2016 .4262$

\begin{abstract}
The current study describes a case of adenosquamous carcinoma originating from a thyroglossal duct cyst (TGDC). A 77-year-old man presented with an asymptomatic mass in the left mid-neck, which was soft and mobile on palpation. Fine-needle aspiration was performed, but cytology did not detect any malignant cells. Computed tomography demonstrated a single cystic lesion in the left lobe of the thyroid gland; therefore, surgery was performed on the suspected thyroid cyst. However, it was identified intraoperatively that the lesion was separated from the thyroid gland and instead adhered to an additional hyoid bone; therefore, the Sistrunk procedure was performed. Histopathological examination of the resected tumor confirmed the diagnosis of adenosquamous carcinoma originating from a TGDC. Carcinoma arising from a TGDC is rare, and accounts for $1 \%$ of all TGDC cases. The most common subtype of carcinoma associated with TGDC is papillary carcinoma, whilst adenosquamous carcinoma developing from a TGDC is extremely rare, with only one case currently reported in the literature. Although a consensus for the management of this disease has not yet been established, adequate surgical excision with long-term follow-up is currently the preferred treatment.
\end{abstract}

\section{Introduction}

A thyroglossal duct cyst (TGDC) is a common congenital anomaly that primarily consists of benign lesions (1). Carcinoma arising from a TGDC is rare, accounting for $\sim 1 \%$ of all TGDC cases (2). TGDC associated with carcinoma typically presents as a painless mass in the mid-neck (2). It is often difficult to differentiate TGDC carcinoma from other diseases based on clinical presentation alone; thus, pathological

Correspondence to: Dr Yu-Sung Chang, Department of Otolaryngology, Kaohsiung Veterans General Hospital, 386 Ta-Chung 1st Road, Kaohsiung 813, Taiwan, R.O.C.

E-mail: fuwadaichi@gmail.com

Key words: adenosquamous carcinoma, thyroglossal duct cyst, diagnosis, treatment analysis is required for an accurate diagnosis. The most common subtype of carcinoma arising from a TGDC is papillary carcinoma, followed by the less prevalent squamous cell carcinoma (2). TGDC associated with adenosquamous carcinoma is extremely rare, and only one case has been reported in literature to date (3). The current case describes a patient with adenosquamous carcinoma arising in a TGDC, which presented as a lateral neck mass, and is followed by a discussion of the diagnosis and subsequent management of the disease.

\section{Case report}

A 77-year-old man presented to the Department of Otolaryngology of Kaohsiung Veterans General Hospital (Kaohsiung, Taiwan) with a large mass in the left mid-neck. The patient visited a regional hospital 2 months prior to admission due to hoarseness. On physical examination, a painless neck mass was detected incidentally, and the patient was subsequently referred to the Department of Otolaryngology of Kaohsiung Veterans General Hospital.

Physical examination identified a soft, ballotable, mobile mass that measured $\sim 5 \times 6 \mathrm{~cm}$ in size. A fiberoptic endoscopy was performed, but no lesion was observed in the upper aerodigestive tract, except for one small polyp-like lesion in the anterior third of the right vocal cord. Fine-needle aspiration (FNA) was performed on the neck mass, and $\sim 30 \mathrm{ml}$ of aspirated brown fluid was sent for cytology. A large number of histiocytes were reported, without any presence of malignant cells. Computed tomography (CT; Brilliance 64 Slice CT; Philips, Amsterdam, Netherlands) demonstrated a cystic lesion of 41x34 mm in size, which was located in the left lobe of the thyroid gland (Fig. 1), thus a thyroid cyst was suspected. An additional hyoid bone, which was adjacent to the upper side of the cystic lesion, was also identified by CT.

The patient was admitted to the hospital, and initially underwent microscopic laryngeal surgery to remove the right vocal polyp. The vocal lesion showed edema, proliferation of fibroblasts, hyalinization of stroma and dilated blood vessels in the lamina propia. The vocal lesion was covered with an intact squamous epithelium and had no evidence of neoplasia. Therefore, vocal polyp was diagnosed.

A subtotal thyroidectomy of the left lobe of the thyroid gland was performed 1 week later. During surgery, it was 
A

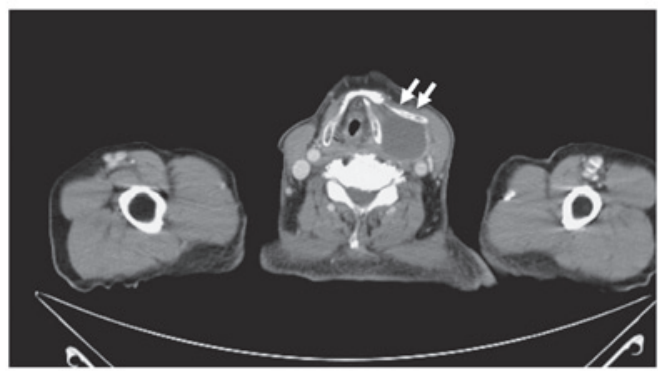

B

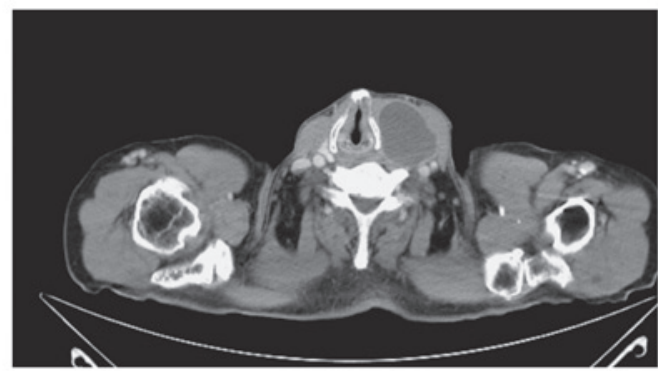

Figure 1. Head and neck computed tomography demonstrated (A) a single hypodense lesion in the left neck, which adhered to an additional hyoid bone (white arrows). (B) The cystic lesion was $\sim 41 \times 34 \mathrm{~mm}$ in size, and due to its position, it was considered to have originated from the left lobe of the thyroid gland.

A

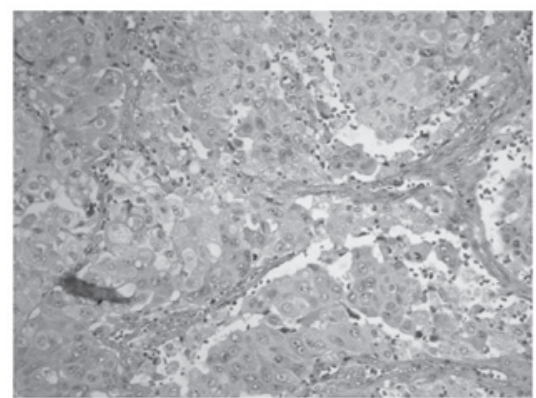

C

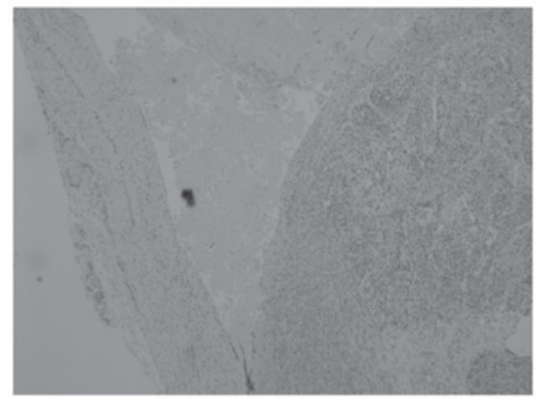

B

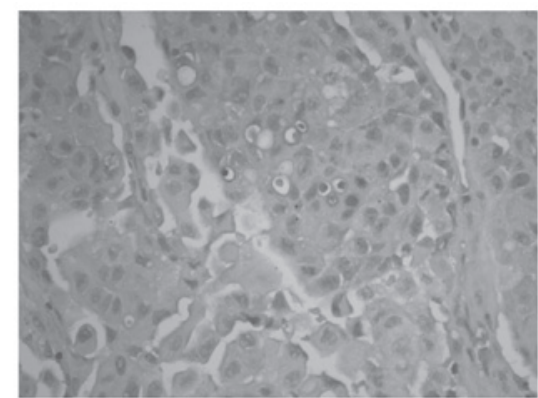

D

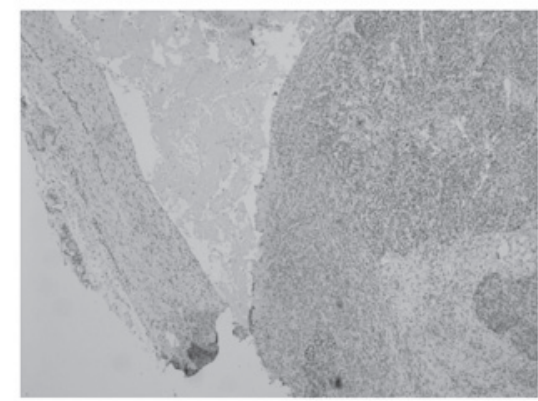

Figure 2. Histological analysis of the resected cystic lesion (hematoyxlin and eosin stain). (A) The tumor was composed of poorly-differentiated, neoplastic squamous cells, which contained hyperchromatic and pleomorphic nuclei, and exhibited a glandular formation (magnification, x200). (B) The tumor tissue contained mucin droplets (staining, mucicarmine; magnification, x200). The tumor cells were negative for (C) paired box 8 and (D) thyroid transcription factor-1 immunostains, which excluded a diagnosis of thyroid carcinoma (magnification, $\mathrm{x} 40$ ).

observed that the cystic lesion was separated from the left lobe of the thyroid gland. The lesion had clear margins to the surrounding tissues, however, the upper side of the cyst was adhered to the additional hyoid bone. Therefore, the cyst and the additional hyoid bone were completely removed. There were a small number of dark, firm lymph nodes, with the largest one (measuring $1.3 \mathrm{~cm}$ in size) located in the left levels II and III of the neck. Frozen section analysis of the lymph nodes revealed reactive hyperplasia. Briefly, tissues were embedded in optimal cutting temperature compound (Shandon Cryomatrix; Thermo Fisher Scientific Inc., Waltham, MA , USA) and frozen rapidly. They were sectioned with a thickness of $5 \mu \mathrm{m}$ using a HM400 microtome (Microm UK Ltd., Bicester, UK) and stained with hematoxylin and eosin (Scharlab SL, Barcelona, Spain). Microscopic examination revealed enlarged lymph nodes with hyperplasia of germinal centers, polymorphous follicles and intact mantle zones. Reactive hyperplasia was diagnosed accordingly.

Gross examination of the cyst detected a high content of a brown fluid. Microscopically (Eclipse 50i; Nikon Corporation, Tokyo, Japan), the cyst was lined by squamous and respiratory epithelium. Poorly-differentiated, neoplastic squamous cells were identified in a glandular formation, which displayed hyperchromatic and pleomorphic nuclei (Fig. 2). Mucin secretion was identified by mucicarmine histochemical staining. Briefly, the tissues were fixed in buffered formalin (Tonyar Biotech, Inc., Taoyuan, Taiwan) and then embedded in paraffin (Leica Biosystems, Wetzlar, Germany). They were sectioned with the thickness of $4 \mu \mathrm{m}$ and stained with hematoxylin and eosin stain (Scharlab SL). Immunostaining was performed for paired box 8 (PAX-8; clone, ZR-1; rabbit anti-human monoclonal antibody; dilution, 1:50; catalog no., Z2202; Zeta Corporation, Arcadia, CA, USA) and thyroid transcription factor-1 (TTF-1; clone, SPT24; mouse anti-human monoclonal antibody; dilution, 1:200; catalog no., TTF-1-L-CE; Novocastra; Leica Microsystems GmbH, Wetzlar, Germany). The tumor cells were negative for PAX-8 and TTF-1 immunostaining.

Based on the aforementioned analyses, the final diagnosis was confirmed as adenosquamous carcinoma arising from a TGDC. Due to the old age of the patient and the large size of the tumor, a total thyroidectomy was performed 3 weeks 


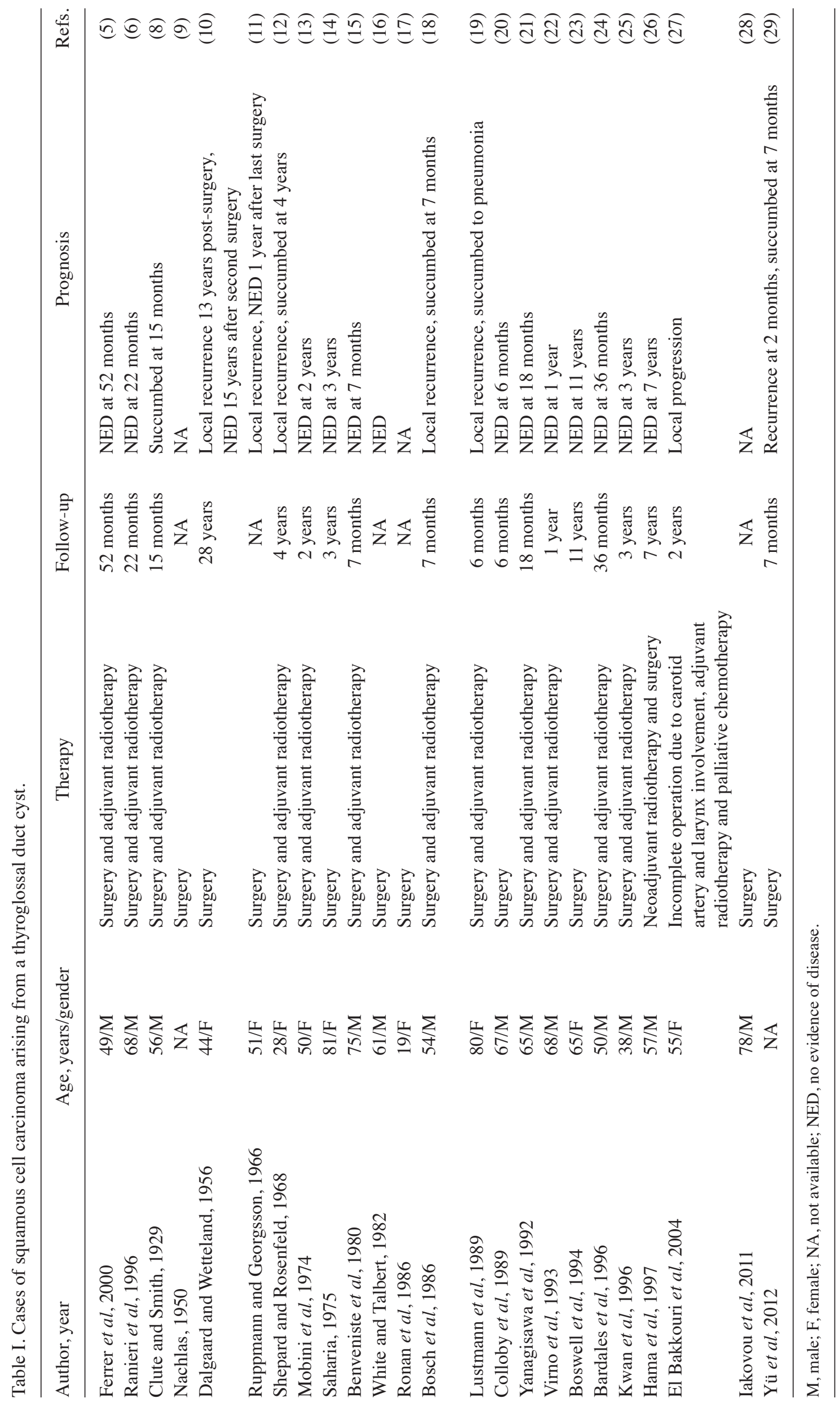


following the first operation. Pathologically, no synchronous neoplastic lesion was detected in the thyroid gland. The patient was subsequently discharged and was followed-up every 2 weeks during the first 2 months and then every 2 months. The patient remains alive without signs of recurrence.

\section{Discussion}

TGDC develops when the tract that forms during the descent of the primordial thyroid gland fails to undergo involution (2). This disease is the most common anomaly of thyroid gland development, with an estimated prevalence of $\sim 7 \%$ (1). The majority of TGDCs are benign. Carcinoma arising from a TGDC is uncommon, and occurs in $\sim 1 \%$ of all TGDC cases (2). Papillary carcinoma is the most common carcinoma to arise from a TGDC, with an incidence of $\sim 80 \%$ of TGDC cases, whilst squamous cell carcinoma is less common, with an incidence of $6 \%$ of all TGDC carcinoma cases (2). Adenosquamous carcinoma associated with a TGDC is extremely rare, with only one case currently reported in the literature (3).

The clinical presentation of TGDC is typically a mobile, painless neck swelling in the midline (4). However, the patient of the current case presented with an asymptomatic neck mass in the lateral neck, which is an atypical location for TGDC. Furthermore, the lesion occurred in proximity to the additional hyoid bone. The symptoms associated with TGDC carcinoma are similar to the those exhibited by a benign TGDC (2). Suspicions of malignancy should be raised if the lesion is hard, fixed, irregular or has undergone recent change (5). However, carcinoma of a TGDC often lacks the aforementioned features, and prior to surgery, it is generally difficult to differentiate such a lesion from a benign TGDC (2). If a reliable diagnosis of TGDC carcinoma can be made preoperatively, a more appropriate surgical intervention may be planned (3). Thus, FNA is considered to be important for preoperative assessment of TGDC (6). However, the present case did not exhibit malignant features, and FNA did not detect any malignant cells, with the final diagnosis being confirmed during pathology.

The treatment of carcinoma arising from a TGDC primarily depends on surgery. The Sistrunk procedure is considered to be adequate for the majority of patients with a clinically and radiologically normal thyroid gland (7). Synchronous neoplastic lesions in the thyroid gland were identified in $\sim 30 \%$ of TGDC carcinoma patients (7). Patel et al (7) suggests that a total thyroidectomy and Sistrunk procedure should be reserved for patients older than 45 years, with a tumor size of $>4 \mathrm{~cm}$, soft tissue extension or with nodal or distant metastasis. Squamous cell carcinoma arising from a TGDC is rare, and is associated with a poor prognosis. Only 24 cases of squamous cell carcinoma arising from a TGDC have currently been reported in the literature (Table I) $(5,6,8-29)$. The median age at presentation is 57.2 years, and the male-to-female ratio is $13 / 9$. The majority of these cases were treated with wide local excision and postoperative radiotherapy. A total of 15 cases reported no evidence of recurrence during follow-up, and 4 cases succumbed to the disease, not including Lustmann et al (19), who succumbed to pneumonia. Local recurrence is common, and was previously reported to occur up to 13 years following the initial treatment $(10,30)$. Therefore, Hanna (30) suggested that wide excision should be performed on localized lesions, and postoperative radiotherapy should be performed on lesions that are larger in size, present extensive nodal disease or exhibit positive surgical margins. To the best of our knowledge, the present case is the second reported case of adenosquamous carcinoma arising from a TGDC. A consensus for the management of this disease has not yet been established, primarily due to the limited available data. A previous study reported that adenosquamous carcinoma of the thyroid gland behaves in a similar aggressive manner to that of anaplastic carcinoma (31). Therefore, it is assumed that patients with TGDC adenosquamous carcinoma may experience a poor prognosis and high local recurrence rate. Due to the old age of the patient and the large tumor size, a more aggressive treatment plan, including the Sistrunk procedure and radical thyroidectomy, was selected for the present case. Pathology demonstrated a clear surgical margin and no malignancy in the thyroid gland or sampled lymph nodes. Therefore, no further treatment was required, with the patient undergoing long-term follow-up alone. Further observation of the clinical course and nature of such tumors is necessary for the improvement of available treatment.

In conclusion, the current study described a rare case of adenosquamous carcinoma arising from a TGDC, which presented as a painless lateral neck mass. The patient lacked clinical features that would indicate a malignant lesion, including a hard, fixed or rapidly growing mass. The diagnosis of this disease is primarily based on pathological findings, resulting in a challenging preoperative planning of adequate surgery. The preferred treatment for TGDC carcinoma is the Sistrunk procedure, whilst other available treatment options include postoperative radiotherapy or a total thyroidectomy (7). Local recurrence of the disease has been reported to occur numerous years following initial treatment; therefore, long-term follow-up is necessary.

The present case showed that the presentation of TGDC adenosquamous carcinoma may be variable and challenging to diagnose by preoperative imaging or fine-needle aspiration cytology. The tumor had distinct margins, which made made the complete resection by the Sistrunk procedure possible. Further studies are required to delineate the long-term prognosis and best treatment strategies for TGDC.

\section{References}

1. Ellis PD and van Nostrand AW: The applied anatomy of thyroglossal tract remnants. Laryngoscope 87: 765-770, 1977.

2. Motamed M and McGlashan JA: Thyroglossal duct carcinoma. Curr Opin Otolaryngol Head Neck Surg 12: 106-109, 2004.

3. Kinoshita N, Abe K, Sainoo Y, Kumagami H, Takahashi H and Hayashi T: Adenosquamous carcinoma arising in a thyroglossal duct cyst: Report of a case. Surg Today 41: 533-536, 2011.

4. Mondin V, Ferlito A, Muzzi E, Silver CE, Fagan JJ, Devaney KO, and Rinaldo A: Thyroglossal duct cyst: Personal experience and literature review. Auris Nasus Larynx 35: 11-25, 2008.

5. Ferrer C, Ferrández A, Dualde D, Rodriguez M, Ferrer E, Pinazo J and Sancho R: Squamous cell carcinoma of the thyroglossal duct cyst: Report of a new case and literature review. J Otolaryngol 29: 311-314, 2000.

6. Ranieri E, D'Andrea MR and Vecchione A: Fine needle aspiration cytology of squamous cell carcinoma arising in a thyroglossal duct cyst. A case report. Acta Cytol 40: 747-750, 1996.

7. Patel SG, Escrig M, Shaha AR, Singh B and Shah JP: Management of well-differentiated thyroid carcinoma presenting within a thyroglossal duct cyst. J Surg Oncol 79: 134-141, 2002.

8. Clute HM and Smith LW: Cancer of the thyroid gland. Arch Surg 18: 1-20, 1929.

9. Nachlas NE: Thyroglossal duct cysts. Ann Otol Rhinol Laryngol 59: 381-390, 1950. 
10. Dalgaard JB and Wetteland P: Aberrant thyroid tissue. II. Thyroglossal anomalies; a follow-up study of 58 cases. Acta Chir Scand 111: 444-455, 1956.

11. Ruppmann E and Georgsson G: Squamous carcinoma of the thyroglossal duct. Ger Med Mon 11: 442-447, 1966.

12. Shepard GH and Rosenfeld L: Carcinoma of thyroglossal duct remnants. Review of the literature and addition of two cases. Am J Surg 116: 125-129, 1968.

13. Mobini J, Krouse TB and Klinghoffer JF: Squamous cell carcinoma arising in a thyroglossal duct cyst. Am Surg 40 290-294, 1974.

14. Saharia PC: Carcinoma arising in thyroglossal duct remnant: Case reports and review of the literature. Br J Surg 62: 689-691, 1975.

15. Benveniste GL, Hunter R and Cook MG: Squamous carcinoma of thyroglossal duct remnants: A case report and review of the literature. Aust N Z J Surg 50: 53-55, 1980.

16. White IL and Talbert WM: Squamous cell carcinoma arising in thyroglossal duct remnant cyst epithelium. Otolaryngol Head Neck Surg 90: 25-31, 1982

17. Ronan SG, Deutsch E and Ghosh L: Thyroglossal duct carcinomas: Light and electron microscopic studies. Head Neck Surg 8: 222-225, 1986.

18. Bosch JL, Kummer EW and Hohmann FR: Carcinoma of the thyroglossal duct. Neth J Surg 38: 36-40, 1986.

19. Lustmann J, Benoliel R and Zeltser R: Squamous cell carcinoma arising in a thyroglossal duct cyst in the tongue. J Oral Maxillofac Surg 47: 81-85, 1989.

20. Colloby PS, Sinha M, Holl-Allen RT and Crocker J: Squamous cell carcinoma in a thyroglossal cyst remnant: A case report and review of the literature. World J Surg 13: 137-139, 1989.

21. Yanagisawa K, Eisen RN and Sasaki CT: Squamous cell carcinoma arising in a thyroglossal duct cyst. Arch Otolaryngol Head Neck Surg 118: 538-541, 1992.
22. Virno VA, Mazzocconi G and Caprio G: Carcinoma a cellule squamose del dotto tireoglosso. Caso Clinico G Chir 14: 351-353, 1993 (In Italian).

23. Boswell WC, Zoller M, Williams JS, Lord SA and Check W: Thyroglossal duct carcinoma. Am Surg 60: 650-655, 1994.

24. Bardales RH, Suhrland MJ, Korourian S, Schaefer RF, Hanna EY and Stanley MW: Cytologic findings in thyroglossal duct carcinoma. Am J Clin Pathol 106: 615-619, 1996.

25. Kwan WB, Liu FF, Banerjee D, Rotstein LE and Tsang RW Concurrent papillary and squamous carcinoma in a thyroglossal duct cyst: A case report. Can J Surg 39: 328-332, 1996.

26. Hama Y, Sugenoya A, Kobayashi S, Itoh N and Amano J: Squamous cell carcinoma arising from thyroglossal duct remnants: Report of a case and results of immunohistochemical studies. Surg Today 27: 1077-1081, 1997.

27. El Bakkouri W, Racy E, Vereecke A, Gauthier A, Quillard J, Bobin S and Portier F: Squamous cell carcinoma in a thyroglossal duct cyst. Ann Otolaryngol Chir Cervicofac 121: 303-305, 2004 (In French).

28. Iakovou I, Konstantinidis I, Doumas A, Nikolaidis V, Karatzas N and Efstratiou I: Squamous cell carcinoma in a thyroglossal duct cyst and 99mTc-MIBI findings. Hell J Nucl Med 14: 62-64, 2011.

29. Yü Y, Wang XL, Xu ZG, Liu SY and Wang JY: Management of thyroglossal duct carcinoma: Report of five cases. Zhonghua Er Bi Yan Hou Tou Jing Wai Ke Za Zhi 47: 1013-1016, 2012 (In Chinese).

30. Hanna E: Squamous cell carcinoma in a thyroglossal duct cyst (TGDC): Clinical presentation, diagnosis, and management. Am J Otolaryngol 17: 353-357, 1996.

31. Nicolaides AR, Rhys Evans P and Fisher C: Adenosquamous carcinoma of the thyroid gland. J Laryngol Otol 103: 978-979, 1989. 